Check for updates

Cite this: RSC Chem. Biol., 2021, 2, 1669

Received 22nd April 2021

Accepted 27th July 2021

DOI: $10.1039 / \mathrm{d} 1 \mathrm{cb} 00089 f$

rsc.li/rsc-chembio

\section{Biodegradable self-assembly micelles significantly enhanced the solubility, biological stability and in vivo antitumor efficacy of Hexylselen $\dagger$}

\author{
Jinzhang Fang, (D) $\ddagger$ Zhao Chen, (D) $\ddagger$ Jun Song, (D) Jinxiu Li, (D) Yunying Han, (D) \\ Wei Hou, (D) Wenxi Wang (D) and Benfang H. Ruan (D)*
}

\begin{abstract}
Glutaminolysis inhibitors have shown early promise in cancer therapeutics. Specifically, kidney-type glutaminase (KGA) has been a long-standing anti-tumor drug target; KGA allosteric inhibitors have attracted great attention due to their superior enzyme specificity and good drug safety profiles. However, the main issue with allosteric inhibitors-including BPTES, CB-839, and the recently developed KGA allosteric and glutamate dehydrogenase (GDH) dual inhibitor, Hexylselen (CPD-3B)-is their low solubility; it leads to limited in vivo efficacy. To optimize their formulation, various delivery carriers were screened in the present study. Soluplus ${ }^{\circledR}$ (SOL), an amphiphilic graft polymer, showed an interesting structure-solubility/activity relationship with Selen molecules containing different middle chain sizes. Among these molecules, the long chain molecule CPD-3B showed 3000-fold increased solubility with SOL, forming well-dispersed and stable micelles 60-80 nm in size. Moreover, CPD-3B@SOL micelles exhibited good metabolic stability in both blood and liver microsomes. These advantages significantly enhanced the bioavailability and in vivo antitumor efficacy of CPD-3B@SOL micelles in the H22 hepatocarcinoma xenograft mouse model. Thus, the current study provided a practical delivery system for allosteric inhibitors of glutaminase, which is one of the bottlenecks of targeting tumor glutaminolysis.
\end{abstract}

\section{Introduction}

Glutaminolysis inhibitors have shown early promise in cancer therapeutics. ${ }^{1}$ Specifically, kidney-type glutaminase (KGA) has been a long-standing anti-tumor drug target; as a KGA active site inhibitor, L-6-diazo-5-oxonorleucine (DON) showed efficacy in cancer treatment early in 1979, but its high toxicity prevented its use as a drug. ${ }^{2}$ The recent development of a pro-drug, JHU083, has brought the DON molecule back under consideration. $^{3}$ KGA allosteric inhibitors have also attracted great attention due to their superior enzyme specificity and good drug safety profiles. However, the main issue with the allosteric inhibitors, such as bis-2-(5-phenylacetamido-1,3,4-thiadiazol-2yl)ethyl sulfide (BPTES) and CB-839 (Telaglenastat), is their low solubility, which leads to limited in vivo efficacy. ${ }^{4,5}$ Efforts have

$I D D \& C B$, College of Pharmaceutical Science \& Collaborative Innovation Center of Yangtze River Delta Region Green Pharmaceuticals, Zhejiang University of Technology, Hangzhou, China. E-mail: ruanbf@yahoo.com, ruanbf@zjut.edu.cn; Fax: +86 571-88871098; Tel: +86-18357023608

$\dagger$ Electronic supplementary information (ESI) available: Determined solubility of CPD-3B in different solvents, the clearance parameters of CPD-3B@SOL micelles in liver microsomes, and the regulated method validation of HPLC analysis and BME derivatization assay for CPD-3B. See DOI: 10.1039/d1cb00089f

\# These authors contributed equally. been made to improve the solubility of CB-839 through medicinal chemistry ${ }^{6}$ and that of BPTES through drug formulation. ${ }^{7}$

Interestingly, the allosteric inhibitor Hexylselen (CPD-3B; Fig. 1A) displayed complete growth inhibition at the cellular level and improved in vivo antitumor efficacy, ${ }^{8,9}$ while CB-839 and BPTES only showed partial growth inhibition at the cellular level. ${ }^{10}$ This was demonstrated by targeting both glutaminase (GLS) and glutamate dehydrogenase (GDH) to completely block glutaminolysis. However, further studies of in vivo efficacy were limited by its poor solubility in various tested solvents (aqueous solution at $1 \mathrm{mg} \mathrm{mL}^{-1}$ in the optimized vehicles).

As a selenazol-3 $(2 \mathrm{H})$-one dimer with a six-carbon straight middle chain, CPD-3B is very flexible; this might cause its low solubility in water and aqueous solvents (Table S1, ESI $\dagger$ ). However, based on the Like Dissolves Like rule, if any appropriate excipients showed similar physical characteristics to $\mathrm{CPD}-3 \mathrm{~B}$, their molecular interaction would likely provide considerable solubility. ${ }^{11}$ Thus, a delivery system formed with amphiphile polymeric materials ${ }^{12}$ might be suitable for CPD-3B.

Due to their high capacity for increasing the solubility, stability, and bioavailability of water-insoluble drugs or even tumor site targeting features, ${ }^{13,14}$ multiple amphiphile 
A

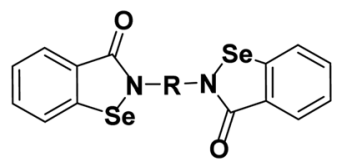

CPD-B Ethaselen $\mathrm{R}=\mathrm{CH}_{2} \mathrm{CH}_{2}$

CPD-1B Propylselen $\mathrm{R}=\mathrm{CH}_{2} \mathrm{CH}_{2} \mathrm{CH}_{2}$

CPD-2B Butaselen $\mathrm{R}=\mathrm{CH}_{2} \mathrm{CH}_{2} \mathrm{CH}_{2} \mathrm{CH}_{2}$

CPD-3B Hexylselen $\mathrm{R}=\mathrm{CH}_{2} \mathrm{CH}_{2} \mathrm{CH}_{2} \mathrm{CH}_{2} \mathrm{CH}_{2} \mathrm{CH}_{2}$
B

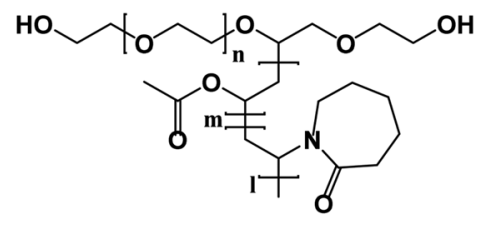

Soluplus $^{\circledR}(\mathrm{SOL})$

Fig. 1 The chemical structures of Selen compounds and the polymer Soluplus ${ }^{\mathbb{R}}$. (A) Selen compounds included CPD-B, CPD-1B, CPD-2B and CPD-3B; (B) Soluplus ${ }^{\mathbb{R}}$ (SOL)

polymeric materials and some modified or responsive polymers ${ }^{15,16}$ especially for chemotherapeutic agents have gained much attention and favor. Some biodegradable copolymers ${ }^{17,18}$ have been extensively investigated for drug delivery systems ${ }^{19}$ and even approved by the Food and Drug Administration (FDA) and used for clinical treatment of carcinoma. ${ }^{20,21}$ The widely used polyvinyl caprolactam-polyvinyl acetate-polyethylene glycol (PVCap-PVA-PEG, $13 \% / 57 \% / 30 \%$, respectively) graft copolymer Soluplus ${ }^{\mathbb{B}}$, also called SOL (Fig. 1B), possesses a low critical micellar concentration (CMC) of $7.6 \mu \mathrm{g} \mathrm{mL}^{-1}$, as well as low toxicity and moderate amphiphilic properties. ${ }^{22}$

Because of its high drug-loading capacity and surfactant property, ${ }^{23}$ SOL shows excellent solubilizing and anti-diluting features for active pharmaceutical ingredients (APIs) in the Biopharmaceutical Classification System (BCS) group II (this system is a valuable tool for guiding drug-like candidates and exploring pharmaceutical formulation in the early stages of development). ${ }^{24}$ SOL can also form nanocarriers to keep the drug saturated in gastrointestinal absorption and sustain its release behavior. ${ }^{25}$

Due to its versatile features, SOL is usually prepared as a solid dispersion, ${ }^{26,27}$ nano-emulsion, ${ }^{28}$ nano-micelle, ${ }^{29-31}$ thermosensitive hydrogel, ${ }^{32,33}$ or transparent film ${ }^{34}$ to increase the solubility and bioavailability of APIs. Recent reports have demonstrated that SOL can form composites with excipients, such as Pluronic ${ }^{\mathbb{R}}$, Solutol HS15, hyaluronic acid-ceramide (HACE), and D- $\alpha$-tocopheryl polyethylene glycol 1000 succinate (Vitamin E TPGS), thereby helping antitumor drugs to avoid toxic agents, achieve higher intestinal epithelial adsorption and better target cancer sites. ${ }^{22}$ Therefore, SOL might formulate a micelle with CPD-3B to overcome the issues of solubility.

This study investigated the solubility and loading efficiency of SOL using selenazol-3(2H)-one dimers (Selen compounds) with different middle chain sizes (Fig. 1A) to explore the structure-solubility/activity relationship (SAR). SOL increased the solubility of CPD-3B by orders of magnitude, and the resulting colloidal and solid states of CPD-3B@SOL were characterized by Malvern dynamic light scattering (DLS), transmission electron microscopy (TEM), and X-ray powder diffraction (XRPD). The CPD-3B@SOL micelles were internalized by tumor cells, as shown under a fluorescence microscope, and they also demonstrated good stability in blood and mouse liver microsomes. Importantly, the CPD-3B@SOL micelles showed significantly improved pharmacokinetics and in vivo antitumor efficacy.

\section{Experiments}

\subsection{Materials and chemical reagents}

The chemical syntheses of CPD-B, CPD-1B, CPD-2B, and CPD-3B have been reported previously. ${ }^{8}$ Soluplus ${ }^{\circledR}$ was gifted by BASF Ltd. (Ludwigshafen, Germany). Hepatoma (H22) and lung cancer (A549) cell lines were purchased from the Shanghai Institute of Biochemistry and Cell Biology of Chinese Academy of Sciences (Shanghai, China). RPMI1640 medium was purchased from M\&C Gene Tech, Inc. (Beijing, China), and fetal bovine serum (FBS) was purchased from Zhejiang Tianhang Biotech, Inc. (Deqing, China). EZMTT detection reagents were obtained from JNF Bioscience, Inc. (Hangzhou, China). ICR mice (SPF) were purchased from the Zhejiang Institute of Medical Science (Hangzhou, China) and treated in compliance with ethical standards. All animal experimental procedures were conducted in conformity with institutional guidelines for the care and use of laboratory animals in Zhejiang University of Technology, Hangzhou, China, and the National Institutes of Health Guide for Care and Use of Laboratory Animals (Publication No. 85-23, revised 1996). ICR/CD1 mouse liver microsomes were purchased from the Research Institute for Liver Diseases, Inc. (Shanghai, China). Coumarin was provided by Meryer Chemical Technology, Inc. (Shanghai, China). $\mathrm{NADPNa}_{2}$ was purchased from Shanghai Yuanye Biotech, Inc. (Shanghai, China). Glucose-6-phosphate (G6P) and G6P dehydrogenase (G6PDH) were purchased from Plant Cell Biotechnology, Inc. (Beijing, China). Chromatographic grade methanol was obtained from Tedia, Inc. (USA), and phosphate buffer saline (PBS) was purchased from Solarbio Inc. (Beijing, China). Milli-Q water (Millipore, Bedford, MA, USA) was used throughout the study, and all other reagents were of analytical grade.

\subsection{Solubility of Selen compounds}

Powders (more than $2 \mathrm{mg}$ per vial) of Selen compounds (CPD-B, $1 \mathrm{~B}, 2 \mathrm{~B}$, and $3 \mathrm{~B}$ ) were treated with distilled water, DMSO, or $1 \%, 5 \%, 10 \%$, or $20 \%$ SOL in distilled water (1 mL each). The resulting mixtures were shaken at $25{ }^{\circ} \mathrm{C}$ for $24 \mathrm{~h}$, and then the supernatants were collected by centrifugation $(10000 \mathrm{rpm}$ 
for $5 \mathrm{~min}$ ) and filtration. This was followed by reversed-phase high-performance liquid chromatography (RP-HPLC) $^{9}$ to quantify the dissolved Selen compounds.

\subsection{Preparation and formulation of Selen@SOL micelles}

The thin film dispersion method was used to prepare Selen@SOL micelles. Briefly, Selen compounds (5 mg) and SOL (50-100 mg) were completely solubilized in a mixture of dichloromethane and ethanol $(1: 4, \mathrm{v} / \mathrm{v})$ or pure methanol (for CPD-B and $2 \mathrm{~B}$ ) by ultrasonication. The organic solvent was then evaporated under a vacuum of $-0.1 \mathrm{MPa}$ at $40-42{ }^{\circ} \mathrm{C}$ until dry. The obtained thin layer was washed and hydrated with sterile physiological saline (0.9\% NaCl solution) to form CPD-3B@SOL micelles. These were stored at $4{ }^{\circ} \mathrm{C}$ and filtered with a $0.45 \mu \mathrm{m}$ acetate fiber membrane (Zhejiang SORFA Medical Plastic, Deqing, China) to remove the undissolved residue. The optimal input ratios of Selen compounds and SOL were screened by determining the stability of the prepared micelles, which was monitored by observing the compound precipitation within $24 \mathrm{~h}$ at room temperature.

\subsection{Encapsulation efficiency and drug loading}

Encapsulation efficiency (EE\%) was measured mainly based on the percentage of the compounds entrapped within the isolated micelles. Because Selen compounds have limited solubility in physiological saline or PBS $\left(\mathrm{pH}=7.4\right.$; lower than $\left.5 \mu \mathrm{g} \mathrm{mL} \mathrm{m}^{-1}\right)$, their concentrations are far lower outside the micelles than inside the micelles. Therefore, prepared Selen@SOL micelles were centrifuged at $3000 \mathrm{rpm}$ for $10 \mathrm{~min}$ to remove visible precipitated compounds and then filtered through a $0.45 \mu \mathrm{m}$ acetate fiber membrane to isolate the invisible Selen compounds. Then, the filtered micelles $(0.2 \mathrm{~mL})$ were disrupted by methanol (10-50 fold) and quantified by RP-HPLC as previously described. ${ }^{10}$ The EE\% and drug loading (DL\%) were calculated using eqn (1) and (2), respectively. All samples were analyzed three times to obtain the mean \pm standard deviation (SD; $n=3$ ).

$$
\mathrm{EE} \%=\frac{\text { Weight of loaded CPD in micelles }}{\text { Weight of input CPD }} \times 100 \%
$$

$\mathrm{DL} \%=\frac{\text { Weight of loaded CPD in micelles }}{\text { Weight of input CPD }+ \text { Weight of input exipient }} \times 100 \%$

\subsection{Particle size and morphology observations}

The average particle size (intensity) and zeta potential $(\zeta)$ of CPD-3B@SOL micelles or blank SOL micelles were measured by DLS using a Malvern system (Nano SZ, MAL1077746, Malvern Instruments, Worcestershire, UK). The polydispersity index (PDI) was determined for assessing the particle size distribution. The morphology of micelles was observed by TEM (JEM-1200EX, NEC, Japan).

\subsection{X-ray powder diffraction}

The crystalline states of CPD-3B, SOL, ground CPD-3B@SOL solid dispersed powders (beneath 200 mesh), and the CPD-3B@SOL physical mixture were determined by XRPD (D2 PHASER, Bruker AXS GMBH, Karlsruhe, Germany). On the disc of a diffractometer, the samples were tiled and exposed to $\mathrm{Cu}-\mathrm{K} \alpha$ radiation under $40 \mathrm{kV}$ and $25 \mathrm{~mA}$ over the $2 \theta$ range of 3-40 degrees, with a scanning speed of 0.03 degree per min. Diffractograms of the fresh CPD-3B@SOL dispersed powders and ones stored at $25{ }^{\circ} \mathrm{C}$ for about 1 year were compared.

\subsection{Storage stability}

The optimized CPD-3B@SOL micelles were stored at $4{ }^{\circ} \mathrm{C}$ for 1 , 5 , and 10 days $(n \geq 3)$. Then, the average particle size, PDI, EE\%, and DL\% were measured to optimize the storage conditions to achieve micelle stability.

\subsection{In vitro release of CPD-3B}

The dialysis method ${ }^{35}$ was used to investigate the in vitro release behavior of CPD-3B from CPD-3B@SOL micelles. In short, 1-2 mL of CPD-3B@SOL micelles prepared with the optimal prescription were loaded into a dialysis bag with a molecular weight cut-off of 8000-14000 Da and placed in $200 \mathrm{~mL}$ of PBS ( $\mathrm{pH}=7.4$ ) mixed with $0.5 \%$ sodium dodecyl sulfate (SDS) as the release medium at $37{ }^{\circ} \mathrm{C}$. The dialyzed bag was magnetically stirred at $100 \mathrm{rpm}$ and continuously incubated at $37{ }^{\circ} \mathrm{C}$ for several days. At predetermined time points, $5 \mathrm{~mL}$ of release medium was withdrawn from the system, and $5 \mathrm{~mL}$ of fresh medium was remedied. The amount of released CPD-3B was quantified by RP-HPLC to produce the release curve. Meanwhile, the CPD-3B vehicle prepared using a previously reported $\operatorname{method}^{9}$ was determined to obtain the comparative CPD-3B release profile.

\subsection{In vitro antiproliferation assay}

The in vitro anti-tumor activity of free CPD-3B (in PBS with $1 \%$ DMSO) and CPD-3B@SOL micelles was evaluated via an EZMTT-based cell proliferation assay using the liver cancer cell line H22 and the non-small cell lung cancer cell line A549. ${ }^{36}$ Briefly, cells $\left(4 \times 10^{3}\right.$ per well $)$ were seeded in a plate for $4 \mathrm{~h}$ and then treated with a series dilution of a CPD-3B DMSO solution (0-10 $\mu \mathrm{M}$ final), CPD-3B@SOL micelles (0-10 $\mu \mathrm{M}$ final), and blank SOL micelles ( $<1 \%$ DMSO final) for 5 days. Viable cells were quantified using the absorbance of optical density at $450 \mathrm{~nm}\left(\mathrm{OD}_{450 \mathrm{~nm}}\right)$ after adding EZMTT reagents for 2-3 h incubation. The inhibition of specimens to tumor cells was calculated using eqn (3). The tests were repeated in triplicate in at least two independent experiments.

$$
\text { Inhibition to tumor cells } \%=\left(1-\frac{\mathrm{OD}_{\text {sample }}-\mathrm{OD}_{\text {blank }}}{\mathrm{OD}_{\text {control }}-\mathrm{OD}_{\text {blank }}}\right) \times 100 \%
$$

\subsection{Cellular uptake of CPD-3B micelles}

In a 96-well plate, H22 hepatoma cells were seeded $(7.5 \times$ $10^{4}$ per well) and grown in RPMI-1640 medium with $10 \%$ FBS in $5 \% \mathrm{CO}_{2}$ incubator at $37{ }^{\circ} \mathrm{C}$. The cells were then treated with free CPD-3B (in 1\% DMSO-PBS buffer), CPD-3B@SOL micelles (30 $\mu \mathrm{M}$ final), or blank SOL micelles in fresh medium for 0.5 , $2,8,12,24$, and 48 hours. At each time point, the cells were collected by centrifugation to remove the culture media. They were then washed once with PBS $(300 \mu \mathrm{L})$, resuspended in 
PBS $(50 \mu \mathrm{L})$ and placed in new wells. The uptake of CPD-3B into $\mathrm{H} 22$ cells was observed based on the fluorescence intensity of CPD-3B in DAPI mode (excitation $=340-360 \mathrm{~nm}$; emission = 450-490 nm) using a fluorescence microscope (Olympus, LB10W-4251, Japan). Meanwhile, the cells were captured in visual light or bright field (BF) mode.

\subsection{In vitro stability in blood}

The stability of the CPD-3B in blood was measured by the $\beta$-mercaptoethanol (BME) derivatization method. ${ }^{37}$ Briefly, fresh heparin-containing mouse blood $(200 \mu \mathrm{L})$ in $1.5 \mathrm{~mL}$ tubes was preheated at $37{ }^{\circ} \mathrm{C}$, then treated with $1 \mathrm{mM}$ of CPD-3B vehicle $^{9}$ ( $5 \mu \mathrm{L}$; the vehicle contained $5 \%$ ethanol, $5 \%$ Tween 80 , $10 \%$ PEG400 and 3\% F68 in PBS buffer and was used in the following experiments) or CPD-3B@SOL micelles $(5 \mu \mathrm{L})$. After gentle mixing, it was incubated in a $37{ }^{\circ} \mathrm{C}$ water bath for $2 \mathrm{~min}$, $5 \mathrm{~min}, 10 \mathrm{~min}, 30 \mathrm{~min}, 1 \mathrm{~h}$, and $2 \mathrm{~h}$. Aliquots of the blood mixtures were derivatized with $1 \mathrm{M}$ of BME $(18 \mu \mathrm{L})$ in $10 \mathrm{mM}$ of $\mathrm{KH}_{2} \mathrm{PO}_{4}$ buffer $(\mathrm{pH}=7.4 ; 100 \mu \mathrm{L})$, using Ebselen ( $1 \mathrm{mM}$ final) as the inner standard. Ethyl acetate $(700 \mu \mathrm{L})$ was used to terminate the reaction $3 \mathrm{~h}$ later and extract the $\mathrm{BME}$ derivatives of CPD-3B, along with the inner standard. The ethyl acetate extracts were dried and redissolved in methanol for HPLC quantitative analysis (detailed analytical data presented in Fig. S1, ESI $\dagger$ ). Samples were tested in triplicate.

\subsection{Stability in liver microsomes}

The stability of CPD-3B in liver microsomes was tested according to the authors' previous report, ${ }^{10}$ using coumarin as the positive control. Briefly, $50 \mu \mathrm{M}$ of CPD-3B vehicle ${ }^{9}$ (CPD-3B@SOL micelles or coumarin; $10 \mu \mathrm{L}$ ) was preincubated with mouse liver microsomes $\left(0.5 \mathrm{mg} \mathrm{mL}{ }^{-1}\right)$ in PBS $(100 \mu \mathrm{L}, \mathrm{pH}=7.4)$ at $37{ }^{\circ} \mathrm{C}$ for $5 \mathrm{~min}$. The reaction was then initiated by the addition of an NADPH-generating system (final: $3 \mathrm{mM} \mathrm{NADPH}, 7.2 \mathrm{mM} \mathrm{MgCl}_{2}, 6 \mathrm{mM}$ G6P, and 3 units of G6PDH). Subsequently, the mixtures were incubated at $37{ }^{\circ} \mathrm{C}$ in a water bath for $5 \mathrm{~min}, 10 \mathrm{~min}, 30 \mathrm{~min}, 60 \mathrm{~min}, 2 \mathrm{~h}$, and $4 \mathrm{~h}$, respectively. Followed by 3 hours of BME derivatization, the inner standard was added to the mixtures and the reaction was terminated by ethyl acetate. The samples were stored at $-20{ }^{\circ} \mathrm{C}$ until RP-HPLC analysis. The obtained half-time life $\left(t_{1 / 2}\right)$ and intrinsic clearance $\left(\mathrm{CL}_{\mathrm{int}}\right)$ were calculated using eqn (4) and (5), respectively.

$$
\begin{gathered}
t_{1 / 2}=-\frac{0.693}{k_{\mathrm{e}}} \\
\mathrm{CL}_{\text {int }}=-k_{\mathrm{e}} \times \frac{1}{C_{\text {microsomal protein }}}
\end{gathered}
$$

In these equations, $k_{\mathrm{e}}$ is the slope of the relationship of $\ln C$ (compound concentration) and $t$ (incubated time), and $C_{\text {microsomal protein }}$ is the input concentration of microsomal protein. Experiments were performed in triplicate to obtain the mean and SD.

\subsection{In vivo pharmacokinetics study}

The in vivo pharmacokinetic studies were performed in male ICR mice weighing approximately $20 \mathrm{~g}$. These mice were randomly divided into four groups $(n=3)$ for each time point and fasted for $12 \mathrm{~h}$ before drug administration with free access to water. The CPD-3B vehicle ${ }^{9}$ and CPD-3B@SOL micelles were intraperitoneally (IP) administered with a dose of $25 \mathrm{mg} \mathrm{kg}^{-1}$ (CPD-3B) and intravenously (IV) by tail vein with $10 \mathrm{mg} \mathrm{kg}^{-1}$. At predetermined intervals $(2 \mathrm{~min}, 5 \mathrm{~min}, 10 \mathrm{~min}, 0.5 \mathrm{~h}, 1 \mathrm{~h}, 2 \mathrm{~h}$, $4 \mathrm{~h}, 8 \mathrm{~h}, 12 \mathrm{~h}$, and $24 \mathrm{~h}$ for IV; $15 \mathrm{~min}, 0.5 \mathrm{~h}, 1 \mathrm{~h}, 2 \mathrm{~h}, 4 \mathrm{~h}, 8 \mathrm{~h}$, $12 \mathrm{~h}$, and $24 \mathrm{~h}$ for IP), aliquots of blood were collected via the orbital venous plexus and allowed to drip freely into the microcollection tube with heparin sodium. Then, the blood samples were treated with the BME derivatization system described in section 2.11 to extract and quantify CPD-3B in the blood. Data from the blood were analyzed for each mouse using DAS 2.0 (DASforeCDM, Shanghai, China). Non-compartmental analysis was performed, and the pharmacokinetic variables $C_{\max }$ (maximal drug concentration; $\mu \mathrm{g} \mathrm{mL}^{-1}$ ), $T_{\max }$ (time for maximal drug concentration; h), and $\mathrm{AUC}_{(0-t)}$ (area under the curve; $\left.\mu \mathrm{g} \mathrm{h} \mathrm{mL} \mathrm{m}^{-1}\right)$ were generated. The elimination half-life $\left(t_{1 / 2 \beta}\right)$ was determined to be $0.693 / k$ (where $k$ is the elimination-rate constant obtained by the equation, $\mathrm{d} C / \mathrm{d} t=-k \mathrm{C}$, in which $C$ is for concentration and $t$ for time). CL was calculated by dividing the dose by the $\mathrm{AUC}_{0-24 \mathrm{~h}}$. The triplicate experimented data were expressed as the mean $\pm \mathrm{SD}$.

\subsection{In vivo antitumor evaluation}

In vivo antitumor activity was evaluated using a xenograft liver cancer H22 mouse model. ICR male mice (approximately $20 \mathrm{~g}$ by weight; 5-7 weeks old) were divided into groups, with 6 mice per group. Diluted liver cancer $\mathrm{H} 22$ ascite tumor cells $(5.0 \times$ $10^{6}$ cells per $\mathrm{mL} ; 0.2 \mathrm{~mL}$ ) were subcutaneously transplanted into the right armpit of each mouse. ${ }^{10}$ After $24 \mathrm{~h}$, the transplanted mice were treated via IV administration with CPD3B@SOL micelles (3 mg mL $\left.{ }^{-1} ; 0.2 \mathrm{~mL}\right)$ and CPD-3B vehicle ${ }^{9}$ $\left(1 \mathrm{mg} \mathrm{mL}{ }^{-1} ; 0.2 \mathrm{~mL}\right.$ ) once daily for 10 consecutive days. PBS vehicle $^{9}$ (also seen in Section 2.11) and blank SOL micelles were IV administered as control vesicles. On day 11, animals in all groups were euthanized humanely to measure their body weight and collect tumors and blood.

\subsection{Data analysis}

All experiments were conducted three times, and all values were expressed as mean \pm SD. Statistical analysis of significance was performed using SPSS 19.0 (SPSS Inc., USA). The differences between two groups were evaluated using the two-tailed Student's $t$-test, and values of $p<0.05\left(^{*}\right), p<0.01\left({ }^{* *}\right)$, and $p<0.001\left({ }^{* *}\right)$ were considered statistically significant and extremely significant, respectively.

\section{Results and discussion}

\subsection{Improved solubility of CPD-3B}

To explore the possible SAR between solubility with SOL and the chain size of a Selen compound, the solubility of the compounds in water or SOL solution was measured using RP-HPLC. Additionally, the CLogPs of Selen compounds were 
Table 1 The CLogPs and solubility in water and Soluplus ${ }^{\circledR}$ solutions of Selen compounds $\left(25 \pm 2{ }^{\circ} \mathrm{C}\right)$

\begin{tabular}{|c|c|c|c|c|c|}
\hline \multicolumn{2}{|l|}{ Physicochemical index } & CPD-B & CPD-1B & CPD-2B & CPD-3B \\
\hline Water solubility $\left(\mu \mathrm{g} \mathrm{mL}^{-1}\right)$ & & $2-3$ & $4-5$ & $1-3$ & $1-2$ \\
\hline Solubility in DMSO $\left(\mathrm{mg} \mathrm{mL}^{-1}\right)$ & & 22 & 29 & 51 & 34 \\
\hline & $5 \%$ & $13.9 \pm 2.1$ & $165 \pm 6$ & $116 \pm 10$ & $272 \pm 22$ \\
\hline & $10 \%$ & $15.2 \pm 0.8$ & $232 \pm 13$ & $246 \pm 9$ & $515 \pm 8$ \\
\hline & $20 \%$ & $48.2 \pm 2.4$ & $300 \pm 17$ & $575 \pm 34$ & $883 \pm 25$ \\
\hline
\end{tabular}

${ }^{a}$ ClogP: the calculated logP by the online program ALogPS 2.1 (http://www.vcclab.org/lab/alogps/). ${ }^{b}$ The concentrations of $1 \%, 5 \%, 10 \%$ and $20 \%$ Soluplus ${ }^{\circledR}$ used were $10 \mathrm{mg} \mathrm{mL}^{-1}, 50 \mathrm{mg} \mathrm{mL}^{-1}, 100 \mathrm{mg} \mathrm{mL}^{-1}, 200 \mathrm{mg} \mathrm{mL}^{-1}$, respectively.

A

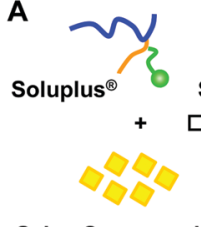

Selen Compound

B

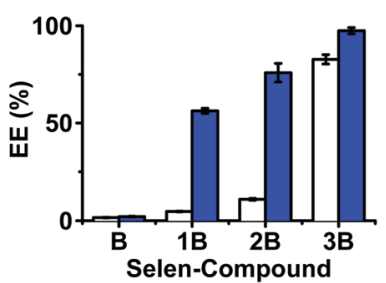

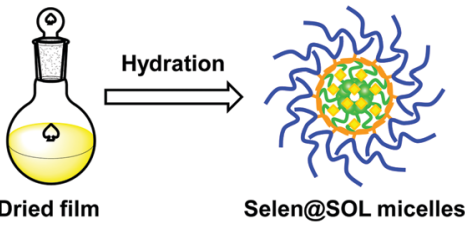

C

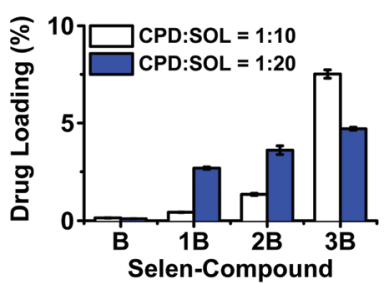

Fig. 2 The preparation of SelenaSOL micelles. (A) The preparation process of SelenaSOL micelles; (B) the encapsulation efficiency (EE) showing the percentage of the enclosed compound in SOL micelles and (C) drug loading (DL) showing the amount of the compound in each Selen@SOL micelle, indicating that a compound: Soluplus ${ }^{\circledR}$ (CPD : SOL) ratio between $1: 10$ and $1: 20$ might be the optimal condition (the input of Selen compound: $2 \mathrm{mg} \mathrm{mL}^{-1}$ per vial).

calculated using the professional platform ALogPS 2.1 (Table 1). The four Selen molecules containing straight 2, 3, 4, or 6-carbon middle chains showed poor solubility in water (1-5 $\mu \mathrm{g} \mathrm{mL}^{-1}$ ), especially CPD-3B which contains a longer middle carbon linker and higher CLogP. This was the case even though CPD-3B solubility in DMSO was good, in the range of about $20-50 \mathrm{mg} \mathrm{mL}^{-1}$. Interestingly, polymeric SOL significantly increased the solubility; the limited SAR showed that the longer the middle chain (from 2 to 6 carbon chains), the better

the solubility by $20 \%$ SOL (solubility from 48 to $882 \mu \mathrm{g} \mathrm{mL} \mathrm{m}^{-1}$ ). For example, with increasing amounts of SOL (from 0 to $20 \%$ ), the solubility of CPD-3B increased nearly 400 times and reached $0.88 \mathrm{mg} \mathrm{mL}^{-1}$. This indicated that the longer middle chain could produce higher hydrophobicity and could strongly interact with SOL; the encapsulation in the lipophilic core of the micellar system significantly enhanced the solubility of Selen compounds in aqueous solution.

\subsection{Preparation and preliminary evaluation of Selen@SOL micelles}

The thin layer dispersion method was used to prepare self-assembled micelles, since SOL significantly enhanced the solubility of Selen compounds (Fig. 2A). As shown in Fig. 2B, when a mass ratio of $1: 10$ (compound:SOL) was used, CPD-B, CPD-1B, and CPD-2B displayed low EE\% $(<10 \%)$ in the SOL micelles. However, when the mass ratio increased to $1: 20$ (compound : SOL), CPD-1B and CPD-2B displayed significantly improved $\mathrm{EE} \%$ and the corresponding DL\% values were in agreement with the $\mathrm{EE} \%$.

CPD-3B showed good interaction with SOL as indicated by greater than $85 \%$ EE\% at compound:SOL ratios of both 1:10 and $1: 20$. Even though 10\% higher EE\% was achieved with a 1:20 compound SOL ratio, double the amount of SOL used resulted in lower DL\% in 1:20 SOL micelles. This demonstrated that SOL showed higher solubilizing and encapsulating capacity for CPD-3B through the preparation methods of the current study so that the resulting CPD-3B@SOL micelles were further optimized and used as the delivery system for cancer treatment.

\subsection{Morphology characterization of CPD-3B@SOL micelles}

For further optimizing the CPD-3B@SOL micelle preparation procedures, we varied the input compound and SOL

Table 2 The particle size, polydispersity index, encapsulation efficiency, and appearance description of CPD-3B@SOL micelles at different mass ratios $(n \geq 3)$

\begin{tabular}{lllllll}
\hline CPD-3B : SOL w/w & $C_{\text {CРD-3в }}\left(\mathrm{mg} \mathrm{mL}^{-1}\right)$ & $C_{\text {SOL }}\left(\mathrm{mg} \mathrm{mL}^{-1}\right)$ & Particle size $(\mathrm{nm})$ & PDI & EE\% & Description \\
\hline $0: 20$ & 0 & 100 & $77.7 \pm 1.1$ & $0.18 \pm 0.02$ & - & Clear, opalescent \\
$1: 10$ & 1 & 10 & $63.4 \pm 1.4$ & $0.05 \pm 0.003$ & $85.1 \pm 1.9$ & Slight precipitation \\
$1: 15$ & 1 & 15 & $65.8 \pm 4.2$ & $0.14 \pm 0.02$ & $95.9 \pm 1.6$ & Clear, opalescent \\
$1: 20$ & 1 & 20 & $64.3 \pm 5.2$ & $0.13 \pm 0.002$ & $95.6 \pm 2.3$ & Clear, opalescent \\
$1: 20$ & 5 & 100 & $77.9 \pm 2.1$ & $0.17 \pm 0.02$ & $94.3 \pm 6.1$ & Clear, opalescent
\end{tabular}

Notes: SOL: Soluplus ${ }^{\circledR}$; w/w: weight of CPD-3B/weight of SOL; CCPD-3B: the input content of CPD-3B; CSOL: the input content of Soluplus ${ }^{\circledR}$; particle size: the average sizes of micelles; PDI: polydispersity index; EE: encapsulation efficiency. 
A

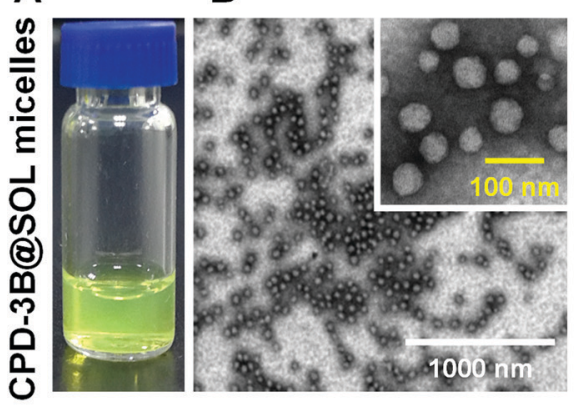

E

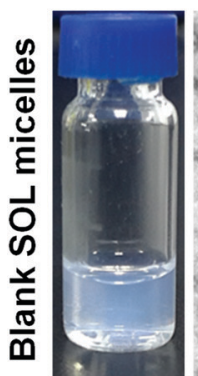

$\mathbf{F}$

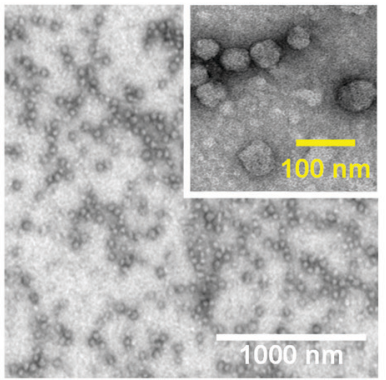

C

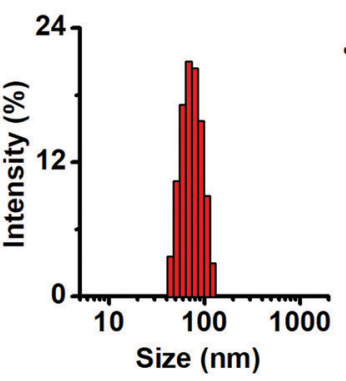

G

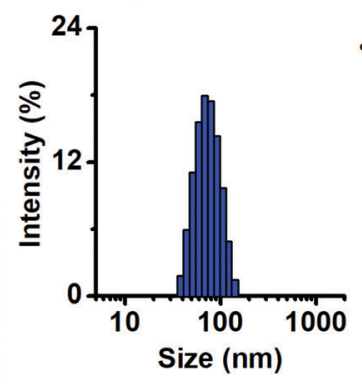

D

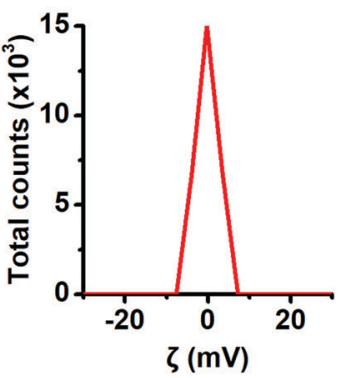

$\mathrm{H}$

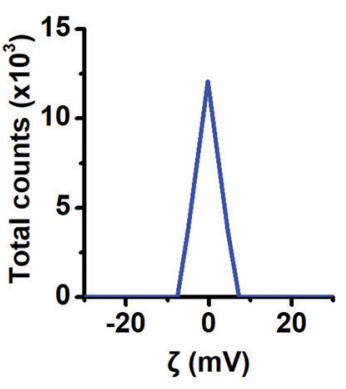

Fig. 3 The particle characterization of CPD-3B@SOL micelles and blank SOL micelles. (A) At $25 \pm 2{ }^{\circ} \mathrm{C}, 5 \mathrm{mg} \mathrm{mL}{ }^{-1} \mathrm{CPD}-3 \mathrm{~B}$ in CPD-3B@SOL micelles $\left(\mathrm{SOL}=100 \mathrm{mg} \mathrm{mL}^{-1}\right.$ ) showed a bright yellow transparent opalescence; (B) TEM images of CPD-3B micelles; (C and D) CPD-3B micelles had average sizes of about 70-80 nm (C) with good distribution and zeta potential close to zero $\mathrm{mV}$ (D). (E) $100 \mathrm{mg} \mathrm{mL}^{-1} \mathrm{SOL}$ micelles had light blue transparent opalescence at $25 \pm 2{ }^{\circ} \mathrm{C}$; (F) images of blank SOL micelles by TEM; ( $G$ and H) average particle sizes at about $70-80 \mathrm{~nm}$ and zeta potential close to zero $\mathrm{mV}$ of blank micelles.

concentration, but kept their ratio between $1: 10$ and $1: 20$. Even though CPD-3B@SOL micelles in both 1:10 and 1:20 achieved more than $85 \%$ (Table 2), when the input of SOL (1\%) decreased, the encapsulating ability of CPD-3B was weakened which led to slight precipitation. When high concentration of SOL was used, the particle size and PDI of the CPD-3B@SOL micelles mildly increased. In Table 2 , when the $10 \%$ (or $100 \mathrm{mg}$ $\mathrm{mL}^{-1}$ ) SOL was used, with or without the CPD-3B, the resulting micelles showed essentially the same sizes. Using 1-2\% SOL thus resulted in smaller sizes at about 60-70 $\mathrm{nm}$ but the EE\% was maintained at $85 \%-96 \%$. Furthermore, the calculated aqueous solubility of CPD-3B reached $4.5 \mathrm{mg} \mathrm{mL}^{-1}$ at a ratio

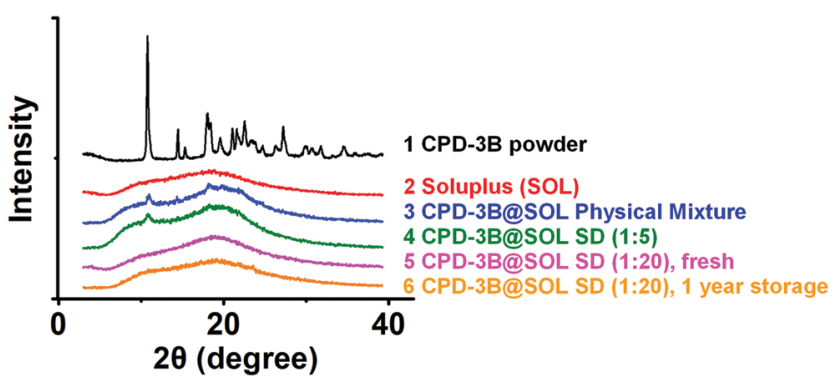

Fig. $4 \quad X$-ray powder diffraction characterization. The tested samples included (1) crystalline CPD-3B, (2) Soluplus ${ }^{\circledR}$ powder, (3) CPD-3B@SOL physical mixture (PM), (4) and (5) fresh CPD-3B@SOL solid dispersion (CPD-3BCSOL SD) at respective mass ratios of $1: 5$ and $1: 20$, and (6) CPD-3BCSOL SD $(\mathrm{w} / \mathrm{w}=1: 20)$ stored for 1 year. of $1: 20$ (CPD-3B:SOL), which was about 3000-fold improved solubility in water.

Because of its low CMC, SOL quickly and spontaneously encapsulated CPD-3B into its hydrophobic core to form micelles after hydration in water or physiological saline. The prepared CPD-3B@SOL micelles with yellow transparent opalescence showed round and oval shapes with good uniformity under TEM (Fig. 3A and B). This was consistent with the results of DLS, which found that the average particle size of CPD-3B@SOL micelles was 60-80 nm and that PDI was less than 0.2 (Fig. 3C). The average potential close to $0 \mathrm{mV}$ indicated that both the CPD-3B@SOL micelles and blank SOL micelles were essentially free of charge (Fig. 3D and $\mathrm{H}$ ). Meanwhile, the particle size and PDI of both the blank micelles and CPD-3B@SOL micelles did not differ, which demonstrated that the self-assembled SOL micelles had excellent micellar compatibility with CPD-3B and could be used for further characterization and biological investigation.

\subsection{X-ray powder diffraction}

To identify the powder state of the CPD-3B@SOL micelles, XRPD was performed. As shown in Fig. 4, crystalline CPD-3B showed a spectrum with multiple peaks, whereas the amorphous SOL solid featured two flat scattering peaks. CPD-3B@SOL (1:5) solid dispersion showed two peaks at $2 \theta$ angles of $10.796^{\circ}$ and $18.049^{\circ}$; these peaks were similar to those of the physical mixture of CPD-3B@SOL, which indicated that inadequate SOL caused incomplete amorphous transformation of CPD-3B from the 
A
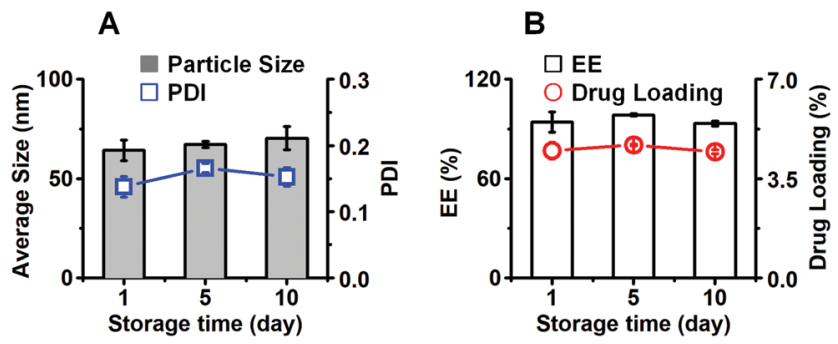

Fig. 5 The storage stability of CPD-3B@SOL micelles. (A) The particle size and PDI, and (B) encapsulation efficiency and drug loading of CPD3B@SOL micelles were tested in 10 days storage at $4{ }^{\circ} \mathrm{C}$.

crystalline state. The mass ratio (CPD-3B:SOL) was then increased to $1: 20$. The peaks at these two $2 \theta$ angles disappeared, and the resulting amorphous peaks were essentially the same as the SOL powder. At a 1:20 mass ratio, the CPD-3B@SOL solid dispersion was stable at room temperature for about one year. Therefore, CPD-3B was verified to be highly compatible and stable with SOL in a solid dispersion.

\subsection{Storage stability}

When the CPD-3B@SOL micelles were stored in a $4{ }^{\circ} \mathrm{C}$ atmosphere protected from light for 10 days (Fig. 5), the micelles maintained an average size of $70-80 \mathrm{~nm}$, a PDI lower than 0.2 , and an average $\mathrm{EE} \%$ and DL\% of $93 \%$ and $4.5 \%$, respectively. No significant difference was observed between the first and the tenth day, which demonstrated that the CPD-3B@SOL micelles were essentially stable under storage conditions.

\subsection{In vitro release}

The in vitro release of free CPD-3B and CPD-3B@SOL micelles was studied in experiments and analyzed via the dialysis method using PBS with $0.5 \%$ SDS (0.5\% SDS-PBS) at $37{ }^{\circ} \mathrm{C}$ as the release medium, and the releasing profiles are shown in Fig. 6. The free CPD-3B exhibited a fast release velocity and reached more than $80 \%$ cumulative release at $24 \mathrm{~h}$, and nearly $100 \%$ over $120 \mathrm{~h}$, while CPD-3B cumulatively released about $70 \%$ at $48 \mathrm{~h}$ and about $80 \%$ at $120 \mathrm{~h}$ from the CPD-3B@SOL micelles (Fig. 6). With the stable hydrophobic core of the polymeric micelles and increased solubility of CPD-3B, the CPD-3B@SOL micelles tended to show a sustained releasing profile, which might be advantageous in in vivo delivery and long circulation.

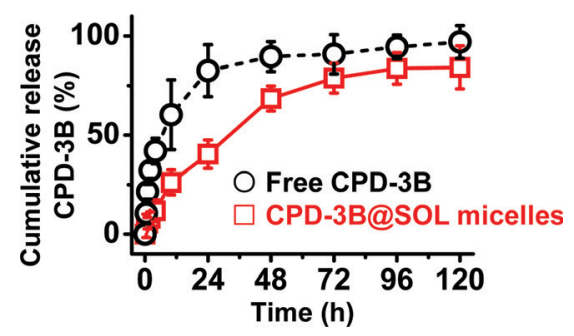

Fig. 6 The in vitro cumulative release profiles of free CPD-3B (5.4 \pm $0.4 \mu \mathrm{g} \mathrm{mL}^{-1}$ final; $100 \%$ release) and CPD-3BaSOL micelles (21 \pm $2.7 \mu \mathrm{g} \mathrm{mL}^{-1}$; final; $100 \%$ release) in $0.5 \%$ SDS-PBS (pH 7.4) at $37^{\circ} \mathrm{C}$.

\subsection{Growth inhibition of CPD-3B@SOL micelles}

Using EZMTT assay to measure the cellular NAD(P)H level, ${ }^{36}$ the inhibitory activities of cancer cell growth by free CPD-3B (in PBS with 1\% DMSO) and CPD-3B@SOL micelles were tested and compared (Fig. 7 and Table S2, ESI $\dagger$ ). Blank SOL micelles did not show inhibition, even at concentrations above $30 \mu \mathrm{M}$, whereas both CPD-3B@SOL micelles and free CPD-3B achieved $100 \%$ inhibition of both $\mathrm{H} 22$ and A549 cell lines. As shown in Fig. 7, the growth inhibition curve showed that the CPD-3B@SOL micelles had slightly weaker (1-2 $\mu \mathrm{M})$ activity than that of free CPD-3B, indicating that micelles did not reduce the potency of the compound. Although the internalization of CPD-3B from free CPD-3B and CPD-3B@SOL micelles into cancer cells might be different, we further investigated its cellular uptake by a CPD-3B compound fluorescence assay.

\subsection{The internalization of CPD-3B@SOL micelles in tumor cells}

To further investigate if there is any difference in cellular uptake between free CPD-3B and CPD-3B@SOL micelles, fluorescence microscopy (DAPI and BF (bright field) modes) was used as a direct, quick, and intact method. The CPD-3B had endogenous emission fluorescence that could be observed under excitation light. As shown in Fig. 8, when the BF and fluorescence images were compared after $24 \mathrm{~h}$ incubation, H22 cells in the blank SOL micelles were basically round, with no fluorescence. When CPD-3B (in PBS with 1\% DMSO) was added, cells with fluorescence emerged, and some cells showed damage. Interestingly, cells in the presence of the CPD-3B@SOL micelles (Fig. 8) showed stronger fluorescence than free CPD-3B, indicating that the CPD-3B@SOL micelles might be able to retain or stay within the cells more efficiently. The CPD-3B@SOL micelles showed 700 RFU fluorescence intensity in the $\mathrm{H} 22$ cell lines which was maintained over 400 RFU throughout the 24 hour incubation, whereas the free CPD-3B peaked at 350 RFU at $2 \mathrm{~h}$ and then decreased with time (Fig. 8M). This demonstrated that CPD-3B could be internalized directly to tumor cells, easily metabolized, and cleared. Moreover, SOL micelles might be able to enhance the stability of CPD-3B, which thus increased its uptake and prolonged its retention in $\mathrm{H} 22$ cells.

\subsection{Stability of CPD-3B@SOL micelles in blood and liver microsomes}

According to previous reports, ${ }^{37}$ Selen compounds, such as Ethaselen and Ebselen, can cross-link with cellular proteins through cystein and selenocystein in plasma and blood. ${ }^{38,39}$ Therefore, BME derivatization is required as a feasible way to isolate the compound. To investigate the stability of CPD-3B in blood and liver microsomes, the BME method was adapted and coupled with RP-HPLC for analysis and quantification (Fig. S1 and $\mathrm{S} 2$, ESI $\dagger$ ).

After $2 \mathrm{~h}$ incubation in blood, the free CPD-3B and CPD3B@SOL micelles showed remaining levels of CPD-3B of $64 \%$ and $80 \%$, respectively, indicating that the CPD-3B@SOL micelles slowed down the metabolism of CPD-3B (Fig. 9A). 

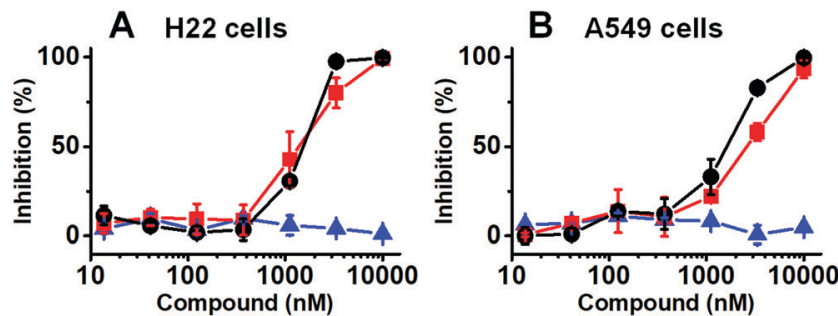

Free CPD-3B

- CPD-3B@SOL micelles

$\triangle$ Blank SOL micelles

Fig. 7 The tumor cell growth inhibition of free CPD-3B, CPD-3B@SOL micelles, and blank SOL micelles. In (A) mouse liver cancer cell lines H22 and (B) human non-small lung cancer cell lines A549, inhibition of the free CPD-3B, CPD-3B@SOL micelles, and blank SOL micelles was compared using EZMTT assay.
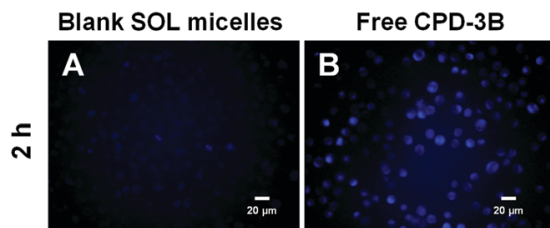

CPD-3B@SOL micelles
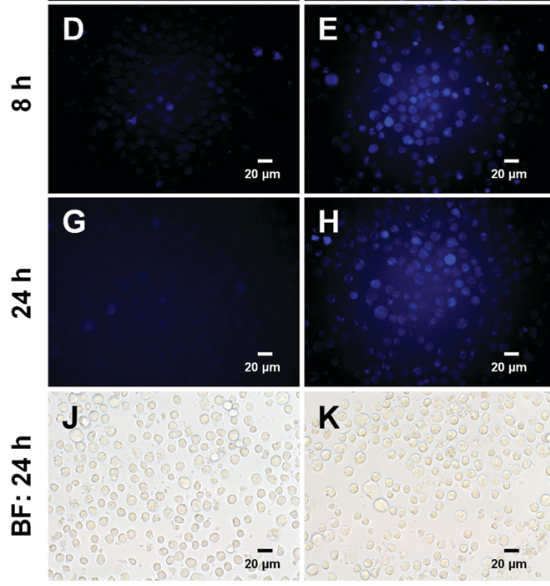

xim

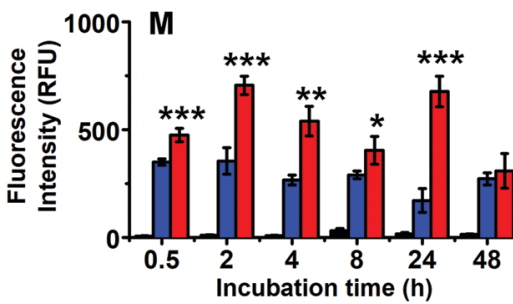

Fig. 8 Soluplus ${ }^{\circledR}$ micelles enhanced the internalization of CPD-3B in tumor cell lines $\mathrm{H} 22$ by fluorescence microscopy. At 2, 8, and $24 \mathrm{~h}$, images of $\mathrm{H} 22$ cells incubated with (A, D, G and J) blank micelles, (B, E, H and $K$ ) free CPD-3B, and (C, F, I and L) CPD-3B@SOL micelles were captured on the modes of DAPI and bright field (BF); (M) during the 48 hours incubation, their fluorescence intensities were quantified and compared $(n \geq 3$; * $p<$ $0.05 ; * *: p<0.01 ; * * *: p<0.001$ ).

After incubation in mouse liver microsomes for $4 \mathrm{~h}, 70 \%$ of the CPD-3B@SOL remained. This was significantly more than free CPD-3B (below 5\%), showing that the micelles helped decrease the $\mathrm{CL}_{\text {int }}$ of CPD-3B (Fig. 9B and Table S3, ESI $\dagger$ ). Therefore, the SOL micelles increased the stability of CPD-3B in blood and liver microsomes and may improve the in vivo delivery and circulation of CPD-3B via blood, decreasing the sensitivity and clearance of $\mathrm{CPD}-3 \mathrm{~B}$ in the liver to realize longer circulation and improved bioavailability.

\subsection{In vivo pharmacokinetics of CPD-3B@SOL micelles}

Pharmacokinetics were performed through IP and IV administration using the CPD-3B vehicle and CPD-3B@SOL micelles to obtain AUC curves and pharmacokinetic parameters (Fig. 10 and Table 3). When IP administered with $25 \mathrm{mg} \mathrm{kg}^{-1}$ dosage, the curves of the CPD-3B vehicle and CPD-3B@SOL micelles showed essentially no difference, while the AUC, $C_{\max }$, and half-life time $\left(t_{1 / 2 \beta}\right)$ increased with slight significance. In the abdomen, the CPD-3B@SOL micelles may have been cleared or adhered by the lymphatic system and ascitic fluid, because 50-700 $\mathrm{nm}$ micelles are distributed and absorbed through the peritoneum and lymphatic duct. ${ }^{40}$ Therefore, IP administration might have caused CPD-3B@SOL micelles to be retained in the peritoneal cavity, thus resulting in no significant enhancement of CPD-3B@SOL micelle concentration in the blood. ${ }^{41}$ This was consistent with early reports that nanoparticles and thermosensitive hydrogels are more suitable for peritoneal tumors. ${ }^{42,43}$

After tail vein injection with dose of $10 \mathrm{mg} \mathrm{kg}^{-1}$ (CPD-3B) for the $\mathrm{CPD}-3 \mathrm{~B}$ vehicle, the $\mathrm{CPD}-3 \mathrm{~B}$ was rarely detectable in the blood $8 \mathrm{~h}$ after the dose, with an AUC of $9.6 \mu \mathrm{g} \mathrm{mL}^{-1} \mathrm{~h}, C_{\max }$ of $6.6 \mu \mathrm{g} \mathrm{mL} \mathrm{m}^{-1}$, and $\mathrm{CL}$ of $1.02 \mathrm{~L} \mathrm{~h}^{-1} \mathrm{~kg}^{-1}$. In contrast, the CPD-3B@SOL micellar system lasted for $24 \mathrm{~h}$ in blood, and the AUC and $C_{\max }$ increased to $48.4 \mu \mathrm{g} \mathrm{mL}^{-1} \mathrm{~h}$ and $68.9 \mu \mathrm{g} \mathrm{mL} \mathrm{m}^{-1}$, respectively. Moreover, due to the high $C_{\max }$ and long maintained concentration at about $1.4 \mu \mathrm{g} \mathrm{mL} \mathrm{m}^{-1}$, the relative bioavailability of the CPD-3B@SOL micelles improved by 504\% of the bioavailability of the CPD-3B vehicle. These results indicated that SOL relatively enhanced the in vivo bioavailability of CPD-3B and prolonged its circulation in the blood, probably due to the significant upgrade in aqueous solubility and blood stability.

\subsection{In vivo tumor depression of CPD-3B micelles}

The in vivo antitumor efficacy of the CPD-3B vehicle and CPD-3B@SOL micelles was compared via the intravenous (IV) administration route. A xenograft $\mathrm{H} 22$ tumor model in ICR mice was utilized for 10 days; the results are shown in Fig. 11. Using the mixture of PEG, Tween 80, and poloxamer 188 as the vehicles, the CPD-3B solution showed very poor solubility. The maximal dosage was $10 \mathrm{mg} \mathrm{kg}^{-1}$, in which the mean tumor weight reduction showed no significance with the blank group via daily IV administration (Fig. 11A and C).

When CPD-3B was encapsulated with SOL, the significant elevated solubility allowed doses of $20 \mathrm{mg} \mathrm{kg}^{-1}$. At the dose of $20 \mathrm{mg} \mathrm{kg}^{-1}$, tumors in the group that received IV-administrated 

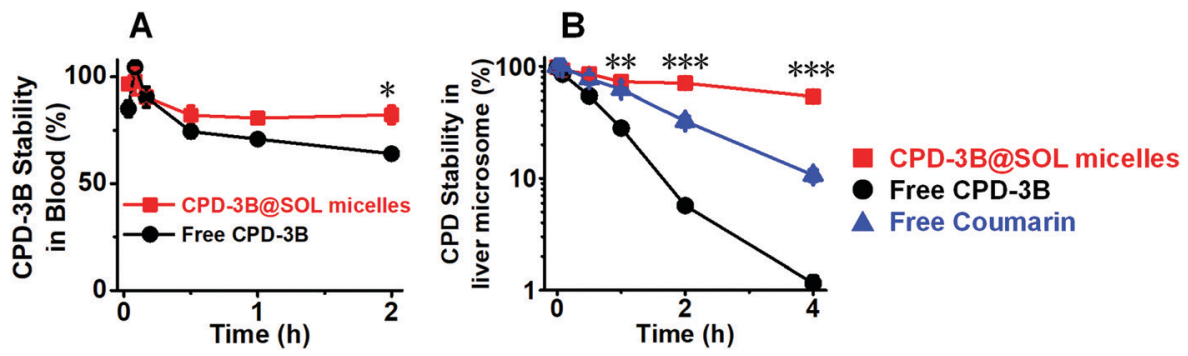

Fig. 9 Soluplus ${ }^{\circledR}$ micelles significantly improved the stability of CPD-3B in blood and liver microsomes. (A) The stability of free CPD-3B and CPD3B@SOL micelles in blood; (B): the stability of Coumarin (positive control), free CPD-3B and CPD-3B@SOL micelles in mouse liver microsomes ( $n \geq 3$; *: $p<0.05 ; * *: p<0.01 ; * * *: p<0.001)$.

A
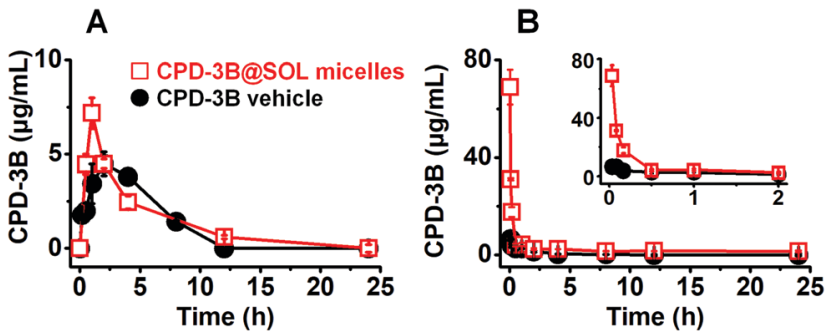

Fig. 10 The area under the curve (concentration-time) of the in vivo pharmacokinetics evaluation. The AUC curves of the CPD-3B vehicle and CPD-3B@SOL micelles were obtained by (A) intraperitoneal injection with $25 \mathrm{mg} \mathrm{kg}^{-1}$; and (B) intravenous administration with $10 \mathrm{mg} \mathrm{kg}^{-1}$.

CPD-3B@SOL micelles were significantly suppressed, with 54\% tumor growth inhibition (TGI) compared to both PBS vehicle and blank SOL micelles (Fig. 11A). Therefore, the limited solubility in regular excipients limited the in vivo efficacy of CPD-3B, while the CPD-3B@SOL micelles achieved much better solubility and stability and better TGI. The improved in vivo efficacy could have benefited from the higher concentrations in the blood and the prolonged time of the CPD-3B micelles. Moreover, no significant difference was found between groups in terms of the weight of the mice (Fig. 11B), which implied that the prescribed CPD-3B@SOL micelles showed minimal toxicity in the animals.
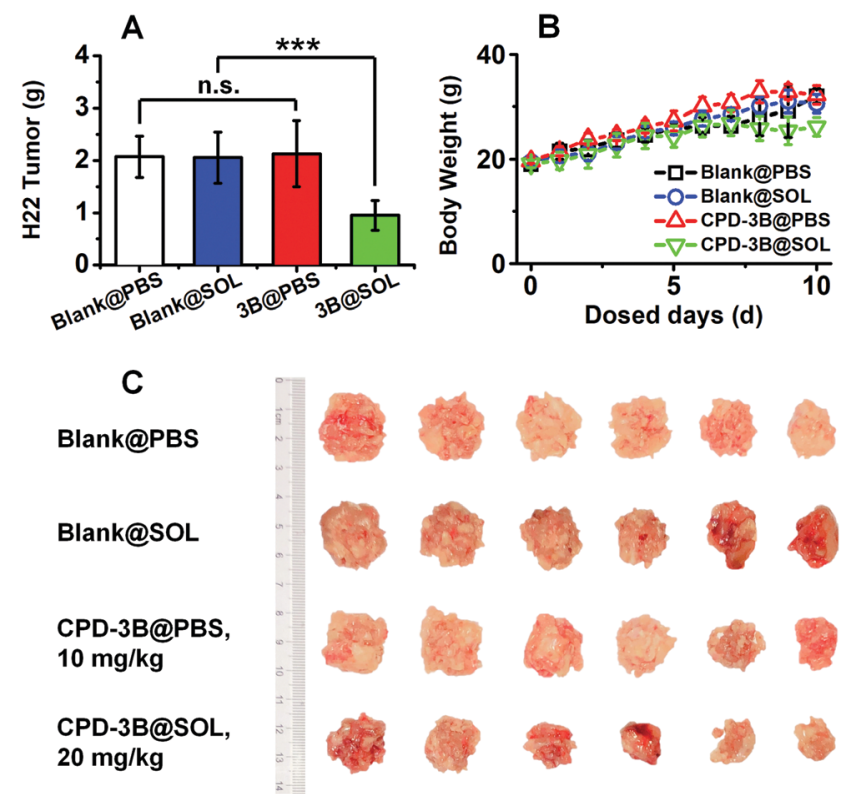

Fig. 11 The growth inhibition of $\mathrm{H} 22$ xenograft tumor via intravenous (IV) administration of the $10 \mathrm{mg} \mathrm{kg}^{-1} \mathrm{CPD}-3 \mathrm{~B}$ vehicle (maximal concentration available) and $20 \mathrm{mg} \mathrm{kg}^{-1} \mathrm{CPD}-3 \mathrm{~B} @ \mathrm{SO} O \mathrm{~L}$ micelles. (A) Hepatoma H22 tumor weight; (B) body weight of mice; and (C) the image of $\mathrm{H} 22$ solid tumors of the mice in all treated groups (IV). (Blank(APBS: PBS vehicle; Blank@SOL: blank Soluplus micelles; 3B@PBS or CPD-3B@PBS: CPD-3B vehicle; $3 B \cong S O L$ or $C P D-3 B C S O L$ : $C P D-3 B @ S O L$ micelles; $S O L$ : Soluplus ${ }^{\mathbb{R}} ; n=6$; n.s.: no significance, $p>0.05$; ${ }^{* \star *}: p<0.001$ ).

Table 3 The in vivo pharmacokinetic parameters of CPD-3B vehicle and CPD-3B@SOL micelles via intraperitoneal and intravenous administration

\begin{tabular}{|c|c|c|c|c|}
\hline \multirow{2}{*}{$\frac{\text { PK parameters }}{\text { Administration }}$} & \multicolumn{2}{|c|}{ CPD-3B vehicle } & \multicolumn{2}{|c|}{ CPD-3B@SOL micelles } \\
\hline & IP & IV & IP & IV \\
\hline Dose $\left(\mathrm{mg} \mathrm{kg}{ }^{-1}\right)$ & 25 & 10 & 25 & 10 \\
\hline $\mathrm{AUC}_{0-24 \mathrm{~h}}\left(\mu \mathrm{g} \mathrm{mL}^{-1} \mathrm{~h}\right)$ & $28.0 \pm 1.4$ & $9.6 \pm 1.1$ & $34.0 \pm 1.2^{*}$ & $48.4 \pm 3.7^{* *}$ \\
\hline$C_{\max }\left(\mu \mathrm{g} \mathrm{mL}{ }^{-1}\right)$ & $4.5 \pm 0.1$ & $6.6 \pm 0.7$ & $7.2 \pm 0.1^{*}$ & $68.9 \pm 7.2^{* *}$ \\
\hline$T_{\max }(\mathrm{h})$ & 2 & 0.033 & 1 & 0.033 \\
\hline$t_{1 / 2 \beta}(\mathrm{h})$ & $2.2 \pm 0.5$ & $1.7 \pm 0.01$ & $4.0 \pm 0.1^{*}$ & $16.4 \pm 4.3^{* * *}$ \\
\hline $\mathrm{CL}\left(\mathrm{L} \mathrm{h}^{-1} \mathrm{~kg}^{-1}\right)$ & $0.87 \pm 0.08$ & $1.02 \pm 0.12$ & $0.71 \pm 0.02$ & $0.10 \pm 0.04$ \\
\hline$F_{\mathrm{r}}(\%)$ & - & - & $121.8 \pm 1.8$ & $504.2 \pm 19.4$ \\
\hline
\end{tabular}

Notes: IP: intraperitoneal; IV: intravenous; $\mathrm{AUC}_{0-24 \mathrm{~h}}$ the area under the curve from $0-24 \mathrm{~h} ; C_{\max }$ : the maximum concentration of CPD in the blood; $T_{\text {max }}$ : the time that CPD concentration in the blood peaked; $t_{1 / 2 \beta}$ : half-life time; CL: the clearance of CPD in the blood; $F_{\mathrm{r}}$ : the relative bioavailability: PK: pharmacokinetic $\left(n \geq 3 ;^{*}: p<0.05 ;^{* *}: p<0.01{ }^{* * *}: p<0.001\right)$ 


\section{Conclusions}

The poor aqueous solubility of various KGA allosteric inhibitors limits their bioavailability and in vivo efficacy. To resolve this bottle-neck, this study explored various drug delivery excipients, such as polyethylene glycols, Tween, poloxamer, cyclodextrin, and SOL (Table S1 (ESI $\dagger$ ) and Table 1). Most excipients showed only limited improvement. For example, the water-soluble hydropropyl- $\beta$-cyclodextrin (HP- $\beta$-CD) has been reported to increase the solubility of Ethaselen significantly by loading a suitably sized molecule into its hydrophobic cave. ${ }^{44}$ However, CPD-3B contains a six-carbon chain in the middle and is more flexible and hydrophobic than Ethaselen, and HP- $\beta$-CD exhibited little solubility enhancement on CPD-3B.

In contrast, SOL significantly enhanced the water solubility of CPD-3B by 3000-fold. As a polyvinyl caprolactam-polyvinyl acetate-polyethylene glycol grafted molecule, SOL has a hydrophobic region that matches the hydrophobic hexyl chain and Selen groups well. Additionally, it has a hydrophilic region that strengthens the hydrophobic core to prevent the compound from precipitation in water; this "like-dissolvelike" feature might contribute a dramatic improvement to solubility. ${ }^{45}$

SAR analysis showed that SOL exhibited a higher capability for dissolving Selen compounds with longer middle chains. The SOL-encapsulated CPD-3B formed stable, nano-sized micelles with good dispersity. Because of their excellent compatibility, the SOL/CPD-3B micelles were stable in aqueous solutions and amorphous solid dispersions. Furthermore, CPD-3B@SOL micelles enhanced the stability of CPD-3B in both mouse blood and liver microsomes and significantly increased the in vivo bioavailability of CPD-3B and pharmacokinetics via IV administration.

Most graft polymeric micelles including SOL micelles have been reported to increase cellular uptake and drug internalization. $^{22,35,45,46}$ In the present study, the fluorescence intensity of CPD-3B showed that CPD-3B@SOL micelles provided higher internalization and longer retention than free CPD-3B in $\mathrm{H} 22$ tumor cells. Perhaps due to the improved pharmacokinetic behaviors and cellular uptake, the CPD-3B@SOL micelles showed improved in vivo antitumor efficacy of CPD-3B in the $\mathrm{H} 22$ liver cancer mouse xenograft model. In contrast, the CPD-3B in PEG and Tween 80 vehicle showed very limited efficacy either by IV or IP administration. ${ }^{9}$

In conclusion, SOL demonstrated an impressive ability to solubilize hydrophobic CPD-3B. This biodegradable SOL not only increased the water solubility of CPD-3B by 3000 times, but also improved the compound's stability in storage, in blood, and under metabolic conditions. In combination with the enhanced cellular uptake, CPD-3B@SOL micelles achieved significant enhancement in in vivo anticancer efficacy for this novel selenium glutaminase inhibitor. The approach of this study provided an advantageous and practical method for improving the in vivo efficacy of potent hydrophobic allosteric glutaminase inhibitors.

\section{Author contributions}

Conception, design and writing, J. F. and B. H. R.; experimental performance and data analysis, J. F., Z. C., J. S., J. L., Y. H., W. H. and W. W. Funding acquisition: B. H. R. and W. H.

\section{Abbreviations}

KGA

DON

BPTES

GLS

GDH

FDA

PVCap-PVA-PEG

CMC

APIs

BCS

HACE

Vitamin E TPGS

Selen compounds

SAR

ClogP

DLS

TEM

XRPD

FBS

G6P

G6PDH

PBS

RP-HPLC

EE

DL

PDI

SOL

SDS

DMSO

EZMTT

BF

BME

$\mathrm{NADPH}$

IV

IP

$\mathrm{AUC}_{0-24 \mathrm{~h}}$

$C_{\max }$

$T_{\max }$

$t_{1 / 2 \beta}$
Kidney-type glutaminase

L-6-Diazo-5-oxonorleucine

Bis-2-(5-phenylacetamido-1,2,4-thiadiazol-

2-yl)ethyl sulfide

Glutaminase

Glutamate dehydrogenase

Food and Drug Administration

Polyvinyl caprolactam-polyvinyl acetatepolyethylene glycol

Critical micellar concentration

Active pharmaceutical ingredients

Biopharmaceutical classification system

Hyaluronic acid-ceramide

D- $\alpha$-Tocopheryl polyethylene glycol 1000 succinate

Selenazol-3(2H)-one dimers

The structure-solubility/activity relationship

Calculated $\log \mathrm{P}$

Dynamic light scattering

Transmission electron microscopy

$\mathrm{X}$-Ray powder diffraction

Fetal bovine serum

Glucose-6-phosphate

Glucose-6-phosphate dehydrogenase

Phosphate buffer saline

Reversed phase high performance liquid chromatography

Encapsulation efficiency

Drug loading

Polydispersity index

Soluplus ${ }^{\circledR}$

Sodium dodecyl sulfate

Dimethyl sulfoxide

2-(3-(2-Methoxy-4-nitrophenyl)-2-(4-

nitrophenyl)-2H-tetrazol-3-ium-5-

yl)benzenesulfonate sodium salt

Bright field

$\beta$-Mercaptoethanol

(Reduced) nicotinamide adenine dinucleotide phosphate

Intravenous administration

Intraperitoneal administration

Area under the curve from 0 to $24 \mathrm{~h}$

The maximum compound concentration in blood

The time that compound concentration peaked in blood

Half-life time 
CL The clearance of CPD in blood

$F_{\mathrm{r}} \quad$ The relative bioavailability

w/w Weight/weight

PM Physical mixture

TGI Tumor growth inhibition

HP- $\beta$-CD Hydropropyl- $\beta$-cyclodextrin

\section{Conflicts of interest}

The authors declare no conflicts of interest in this work.

\section{Acknowledgements}

The authors appreciate the financial support from Start-up funding of Zhejiang University of Technology, China (number 414800129) and Natural Science Foundation of Zhejiang Province/General Project, China (number LY19H300002). We are also thankful for the kind help with transmission electron microscopy by senior engineer Hua Wang from Analysis Center of Agrobiology and Environmental Sciences, Zhejiang University, China.

\section{References}

1 K. Thangavelu, Q. Y. Chong, B. C. Low and J. Sivaraman, Structural basis for the active site inhibition mechanism of human kidney-type glutaminase (KGA), Sci. Rep., 2014, 4, 3827.

2 R. A. Shapiro, V. M. Clark and N. P. Curthoys, Inactivation of rat renal phosphate-dependent glutaminase with 6-diazo-5oxo-l-norleucine. Evidence for interaction at the glutamine binding site, J. Biol. Chem., 1979, 254(8), 2835-2838.

3 R. D. Leone, L. Zhao, J. M. Englert, I.-M. Sun, M.-H. Oh, I.-M. Sun, M. L. Arwood, C. H. Patel, M. Arwood and J. Wen, et al., Glutamine blockade induces divergent metabolic programs to overcome tumor immune evasion, Science, 2019, 366(6468), 1013-1021.

4 S. Krupa, D. V. Ferraris, A. G. Thomas, M. Stathis, B. Duvall, G. Delahanty, J. Alt, R. Rais, C. Rojas and P. Gao, et al., Design, synthesis, and pharmacological evaluation of bis-2-(5phenylacetamido-1,2,4-thiadiazol-2-yl)ethyl sulfide (BPTES) analogs as glutaminase inhibitors., J. Med. Chem., 2012, 55(23), 10551-10563.

5 M. J. Soth, K. Le, M. E. D. Francesco, M. M. Hamilton, G. Liu, J. P. Burke, C. L. Carroll, J. J. Kovacs, J. P. Bardenhagen, C. A. Bristow and M. Cardozo, et al., Discovery of IPN60090, a clinical stage selective glutaminase-1 (GLS-1) inhibitor with excellent pharmacokinetic and physicochemical properties, J. Med. Chem., 2020, 63(21), 12957-12977.

6 J. Li, L. Chen, B. Goyal, G. Laidig, T. F. Stanton and E. B. Sjogren, Heterocyclic inhibitors of glutaminase, US Pat., 9938267B2, 2018.

7 A. Elgogary, Q. Xu, B. Poore, J. Alt, S. C. Zimmermann, L. Zhao, J. Fu, B. Chen, S. Xia and Y. Liu, et al., Combination therapy with BPTES nanoparticles and metformin targets the metabolic heterogeneity of pancreatic cancer, Proc. Natl. Acad. Sci. U. S. A., 2016, 113(36), E5328-E5336.

8 M. Zhu, J. Fang, J. Zhang, Z. Zhang, J. Xie, Y. Yu, J. J. Ruan, Z. Chen, W. Hou and G. Yang, et al., Biomolecular interaction assays identified dual inhibitors of glutaminase and glutamate dehydrogenase that disrupt mitochondrial function and prevent growth of cancer cells, Anal. Chem., 2017, 89(3), 1689-1696.

9 J. J. Ruan, Y. Yu, W. Hou, Z. Chen, J. Fang, J. Zhang, M. Ni, D. Li, S. Lu and J. Rui, et al., Kidney-type glutaminase inhibitor hexylselen selectively kills cancer cells via a three-pronged mechanism, ACS Pharmacol. Transl. Sci., 2019, 2(1), 18-30.

10 Z. Chen, D. Li, N. Xu, J. Fang, Y. Yu, W. Hou, H. Ruan, P. Zhu, R. Ma and S. Lu, et al., Novel 1,3,4-selenadiazolecontaining kidney-type glutaminase inhibitors showed improved cellular uptake and antitumor activity, J. Med. Chem., 2019, 62(2), 589-603.

11 R. Schmid, Recent advances in the description of the structure of water, the hydrophobic effect, and the likedissolves-like rule, Mon. Chem., 2001, 132(11), 1295-1326.

12 M. Valero, F. Castiglione, A. Mele, M. A. da Silva, I. Grillo, G. González-Gaitano and C. A. Dreiss, Competitive and synergistic interactions between polymer micelles, drugs, and cyclodextrins: The importance of drug solubilization locus, Langmuir, 2016, 32(49), 13174-13186.

13 S. S. Das, S. Alkahtani, P. Bharadwaj, M. T. Ansari, M. D. F. ALKahtani, Z. Pang, M. S. Hasnain, A. K. Nayak and T. M. Aminabhavi, Molecular insights and novel approaches for targeting tumor metastasis, Int. J. Pharmaceut., 2020, 585, 119556.

14 W. E. Rudzinski, A. Palacios, A. Ahmed, M. A. Lane and T. M. Aminabhavi, Targeted delivery of small interfering RNA to colon cancer cells using chitosan and PEGylated chitosan nanoparticles, Carbohydr. Polym., 2016, 147, 323-332.

15 R. Gannimani, P. Walvekar, V. R. Naidu, T. M. Aminabhavi and T. Govender, Acetal containing polymers as $\mathrm{pH}-$ responsive nano-drug delivery systems, J. Controlled Release, 2020, 328, 736-761.

16 A. Shah, M. S. Malik, G. S. Khan, E. Nosheen, F. J. Iftikhar, F. A. Khan, S. S. Shukla, M. S. Akhter, H. B. Kraatz and T. M. Aminabhavi, Stimuli-responsive peptide-based biomaterials as drug delivery systems, Chem. Eng. J., 2018, 353, 559-583.

17 C. Englert, J. C. Brendel, T. C. Majdanski, T. Yildirim, S. Schubert, M. Gottschaldt, N. Windhab and U. S. Schubert, Pharmapolymers in the 21st century: Synthetic polymers in drug delivery applications, Prog. Polym. Sci., 2018, 87, 107-164.

18 M. Cagel, F. C. Tesan, E. Bernabeu, M. J. Salgueiro, M. B. Zubillaga, M. A. Moretton and D. A. Chiappetta, Polymeric mixed micelles as nanomedicines: Achievements and perspectives, Eur. J. Pharm. Biopharm., 2017, 113, 211-228.

19 G. S. Kwon, Polymeric micelles for delivery of poorly watersoluble compounds, Crit. Rev. Ther. Drug Carrier Syst., 2003, 20(5), 357-403. 
20 S. Aftab, A. Shah, A. Nadhman, S. Kurbanoglu, S. A. Ozkan, D. D. Dionysiou, S. S. Shukla and T. M. Aminabhavi, Nanomedicine: An effective tool in cancer therapy, Int. J. Pharmaceut., 2018, 540(1-2), 132-149.

21 A. S. Deshmukh, P. N. Chauhan, M. N. Noolvi, K. Chaturvedi, K. Ganguly, S. S. Shukla, M. N. Nadagouda and T. M. Aminabhavi, Polymeric micelles: Basic research to clinical practice, Int. J. Pharmaceut., 2017, 532(1), 249-268.

22 R. Pignatello and R. Corsaro, Polymeric nanomicelles of soluplus as a strategy for enhancing the solubility, bioavailability and efficacy of poorly soluble active compounds, Curr. Nanomed., 2019, 9(3), 184-197.

23 T. Obata, Y. Suzuki, N. Ogawa, I. Kurimoto, H. Yamamoto, T. Furuno, T. Sasaki and M. Tanaka, Improvement of the antitumor activity of poorly soluble sapacitabine (CS-682) by using Soluplus as a surfactant, Biol. Pharm. Bull., 2014, 37(5), 802-807.

24 N. Sachan, A. Bhattacharya, S. Pushkar and A. Mishra, Biopharmaceutical classification system: A strategic tool for oral drug delivery technology, Asian J. Pharm., 2009, 3(2), 76-81.

25 Q. Lin, Y. Fu, J. Li, M. K. Qu, L. Deng, T. Gong and Z. Zhang, A (polyvinyl caprolactam-polyvinyl acetate-polyethylene glycol graft copolymer)-dispersed sustained-release tablet for imperialine to simultaneously prolong the drug release and improve the oral bioavailability, Eur. J. Pharm. Sci., 2015, 79, 44-52.

26 A. Singh, A. Bharati, P. Frederiks, O. Verkinderen, B. Goderis, R. Cardinaels, P. Moldenaers, J. Van Humbeeck and G. Van den Mooter, Effect of compression on the molecular arrangement of Itraconazole-Soluplus solid dispersions: Induction of liquid crystals or exacerbation of phase separation?, Mol. Pharmaceutics, 2016, 13(6), 1879-1893.

27 Y. Zhang, Y. Liu, Y. Luo, Q. Yao, Y. Zhong, B. Tian and $\mathrm{X}$. Tang, Extruded Soluplus/SIM as an oral delivery system: Characterization, interactions, in vitro and in vivo evaluations, Drug Delivery, 2016, 23(6), 1902-1911.

28 G. Quan, B. Niu, V. Singh, Y. Zhou, C. Y. Wu, X. Pan and H. Wu, Supersaturable solid self-microemulsifying drug delivery system: precipitation inhibition and bioavailability enhancement, Int. J. Nanomed., 2017, 12, 8801-8811.

29 Z. Ke, Z. Zhang, H. Wu, X. Jia and Y. Wang, Optimization and evaluation of oridonin-loaded Soluplus $(R)$-Pluronic P105 mixed micelles for oral administration, Int. J. Pharm., 2017, 518(1-2), 193-202.

30 Y. Zhong, G. Jing, B. Tian, H. Huang, Y. Zhang, J. Gou, $\mathrm{X}$. Tang, H. He and Y. Wang, Supersaturation induced by Itraconazole/Soluplus (R) micelles provided high GI absorption in vivo, Asian J. Pharm. Sci., 2016, 11(2), 255-264.

31 I. Salah, Abou Shamat, M. Cook and M. T. Soluplus, solutions as thermothickening materials for topical drug delivery, J. Appl. Polym. Sci., 2019, 136(1), 1-9.

32 H. Wu, K. Wang, H. Wang, F. Chen, W. Huang, Y. Chen, J. Chen, J. Tao, X. Wen and S. Xiong, Novel self-assembled tacrolimus nanoparticles cross-linking thermosensitive hydrogels for local rheumatoid arthritis therapy, Colloids Surf., B, 2017, 149, 97-104.

33 A. Salawi and S. Nazzal, The physiochemical, mechanical, and adhesive properties of solvent-cast vitamin ESoluplus(R) films, Int. J. Pharm., 2018, 552(1-2), 378-387.

34 S. Y. Lee, J.-J. Lee, J.-H. Park, J.-Y. Lee, S.-H. Ko, J.-S. Shim, J. Lee, M. Y. Heo, D.-D. Kim and H.-J. Cho, Electrosprayed nanocomposites based on hyaluronic acid derivative and soluplus for tumor-targeted drug delivery, Colloids Surf., B, 2016, 145, 267-274.

35 Y. Su, K. Wang, Y. Li, W. Song, Y. Xin, W. Zhao, J. Tian, L. Ren and L. Lu, Sorafenib-loaded polymeric micelles as passive targeting therapeutic agents for hepatocellular carcinoma therapy, Nanomedicine, 2018, 13(9), 1009-1023.

36 W. Zhang, M. Zhu, F. Wang, D. Cao, J. J. Ruan, W. Su and B. H. Ruan, Mono-sulfonated tetrazolium salt based NAD(P)H detection reagents suitable for dehydrogenase and real-time cell viability assays, Anal. Biochem., 2016, 509, 33-40.

37 H. Y. Zhou, G. F. Dou, Z. Y. Meng, Y. Q. Lou and G. L. Zhang, High performance liquid chromatographic determination of 1.2-[bis(1,2-benzisoselenazolone-3(2H)-ketone)]-ethane (BBSKE), a novel organoselenium compound, in dog plasma using pre-column derivatization and its application in pharmacokinetic study, J. Chromatogr., B, 2007, 852(1-2), 617-624.

38 M. J. Capper, G. S. A. Wright, L. Barbieri, E. Luchinat, E. Mercatelli, L. McAlary, J. J. Yerbury, S. V. Antonyuk, L. Banci and S. S. Hasnain, et al., The cysteine-reactive small molecule Ebselen facilitates effective sod1 maturation, Nat. Commun., 2018, 9(1), 1693.

39 A. C. Terentis, M. Freewan, T. S. Sempértegui Plaza, M. J. Raftery, R. Stocker and S. R. Thomas, The selenazal drug ebselen potently inhibits indoleamine 2,3-dioxygenase by targeting enzyme cysteine residues, Biochemistry, 2010, 49(3), 591-600.

40 W. J. Shih, J. J. Coupal and H. L. Chia, Communication between peritoneal cavity and mediastinal lymph nodes demonstrated by Tc-99m albumin nanocolloid intraperitoneal injection, Proc. Natl. Sci. Counc. Repub. China B, 1993, 17(3), 103-105.

41 Q. Fu, D. Hargrove and X. Lu, Improving paclitaxel pharmacokinetics by using tumor-specific mesoporous silica nanoparticles with intraperitoneal delivery, Nanomedicine, 2016, 12(7), 1951-1959.

42 H. Cho, T. C. Lai and G. S. Kwon, Poly(ethylene glycol)block-poly(e-caprolactone) micelles for combination drug delivery: Evaluation of paclitaxel, cyclopamine and gossypol in intraperitoneal xenograft models of ovarian cancer, J. Controlled Release, 2013, 166(1), 1-9.

43 W. Zhang, T. Cui, L. Liu, Q. Wu, L. Sun, L. Li, N. Wang and C. Gong, Improving anti-tumor activity of curcumin by polymeric micelles in thermosensitive hydrogel system in colorectal peritoneal carcinomatosis model, J. Biomed. Nanotechnol., 2015, 11(7), 1173-1182.

44 H.-M. Cui and F.-L. Wu, Pharmacokinetics of Eb and its hydroxypropyl- $\beta$-cyclodextrin inclusion complex in rats, J. Chinese Pharm. Sci., 2010, 19, 52-58. 
45 S. Kim, Y. Shi, J. Y. Kim, K. Park and J.-X. Cheng, Overcoming the barriers in micellar drug delivery: Loading efficiency, in vivo stability, and micelle-cell interaction, Expert Opin. Drug Delivery, 2010, 7(1), 49-62.
46 C. Deng, Y. Jiang, R. Cheng, F. Meng and Z. Zhong, Biodegradable polymeric micelles for targeted and controlled anticancer drug delivery: Promises, progress and prospects, Nano Today, 2012, 7, 467-480. 